\title{
Javaneraffen versus Akazienmonokulturen auf Sumatra
}

\author{
HERW IG ZAHORKA
}

\begin{abstract}
Many industrial tree plantations for pulp and paper production have been established on the island of Sumatra within the past two decades. Large surfaces of pristine tropical rainforest were cleared for this purpose. Hordes of long-tailed macaques have discovered acacia monocultures as a welcome alternative diet, were they feed on leaves and bark, in place of their former staple of diverse forest fruit. The damage done to the stems of the acacia trees makes them susceptible to fungal infections and the trees ultimately die. The high demand for industrial pulpwood can lead to further exploitation of the remaining pristine rainforests.
\end{abstract}

\section{Zusammenfassung}

Auf Sumatra wurden in den letzten beiden Jahrzenten viele Nutzholzplantagen für die Zellstoff- und Papierproduktion angelegt. Große Flächen des tropischen Regenwaldes wurden dafür gerodet. Javaneraffen fallen nun in großen Gruppen in Akazienmonokulturen ein, um sich statt von Waldfrüchten von Blättern und Rinde der Akazien zu ernähren. Von Affen geschälte Akazien werden von Pilzen befallen und sterben ab. Da der große Holzbedarf der Konzerne wegen dieser Verluste weniger stark aus Plantagen gedeckt werden kann, besteht die Gefahr, dass vermehrt im Regenwald Holz geschlagen wird.

\section{Großer Holzbedarf der Papierindustrie} Auf der indonesischen Insel Sumatra wurden in den 1990er-Jahren etwa 800000 Hektar Regenwald abgeholzt, um Plantagen mit schnellwüchsigen Nutzhölzern für die Zellstoff- und Papierindustrie anzulegen. Mittlerweile hat sich

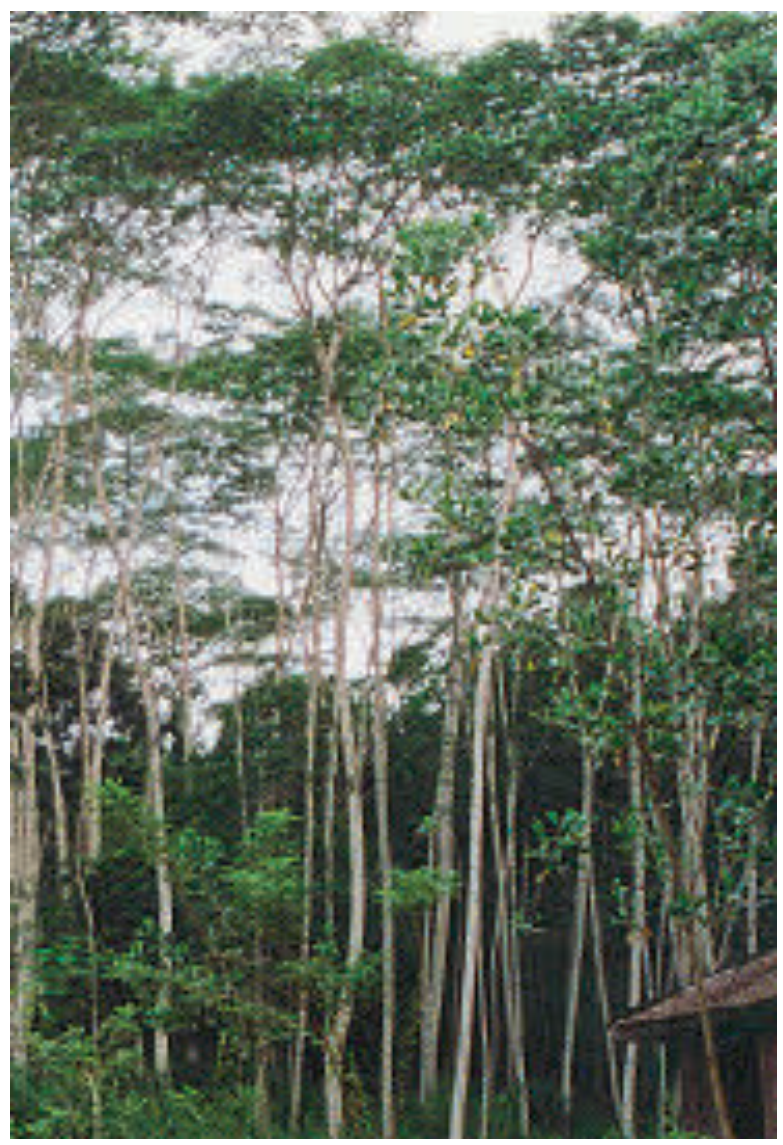

Indonesien zu einem der weltweit größten Papier- und Zellstoffproduzenten entwickelt. Der Holzbedarf ist immens. Um den riesigen Bedarf zu decken, wurden nicht nur wertvolle Regenwaldgebiete für die Anlagen der Plantagen geopfert; noch immer wird im Regenwald,

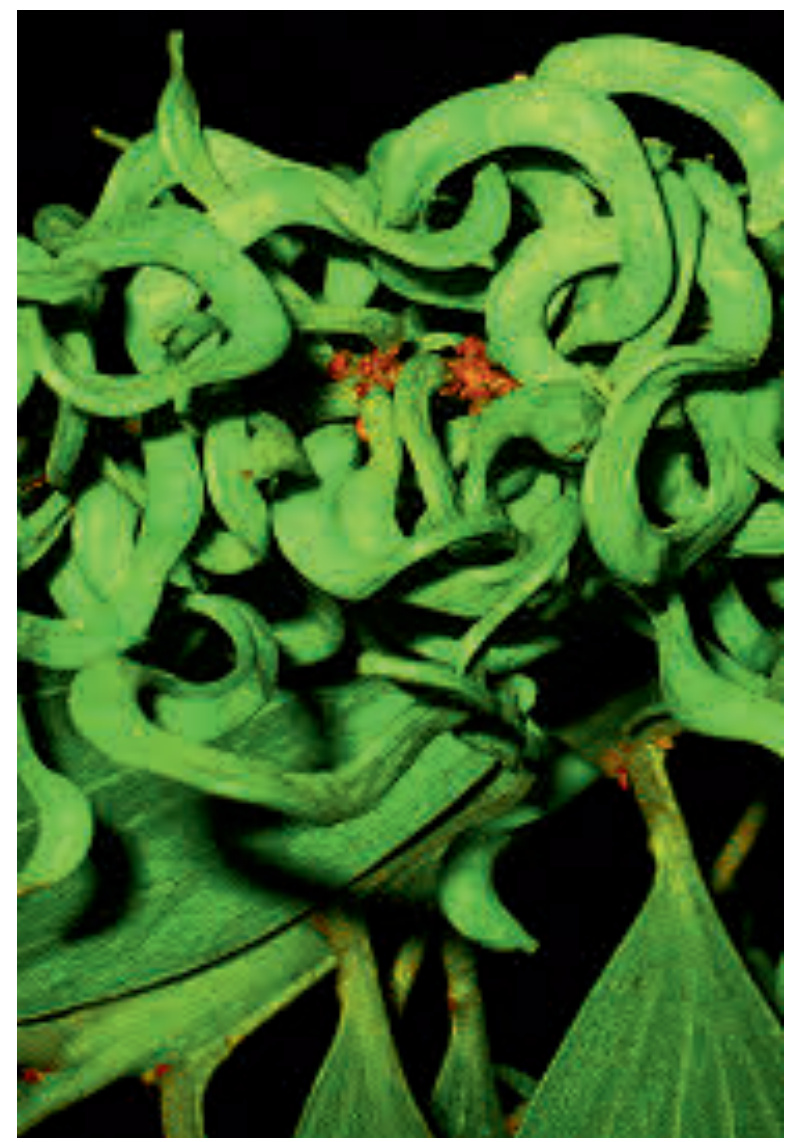




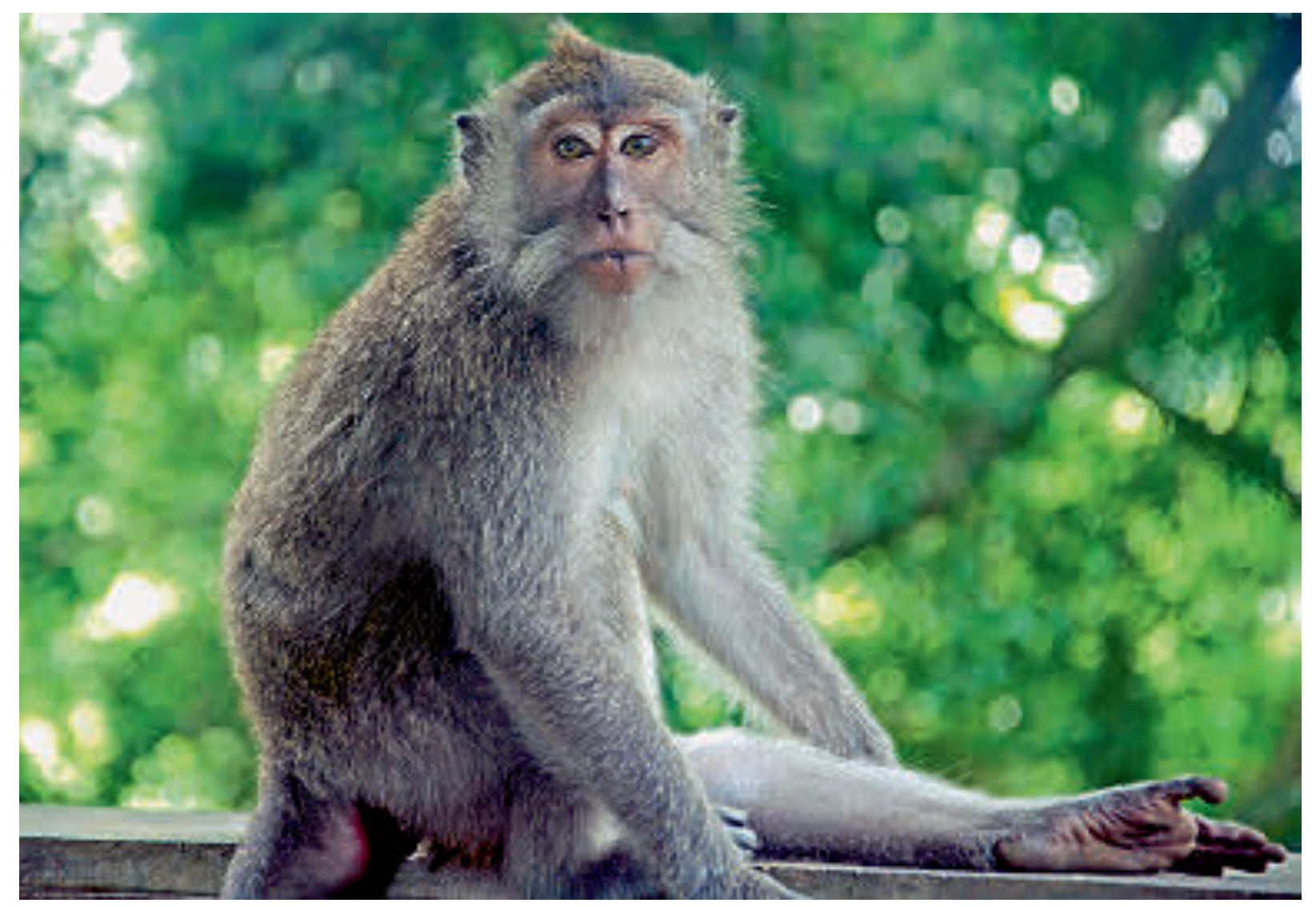

selbst in geschützten Gebieten bis hin zu Nationalparks, illegal Holz für die Papierherstellung eingeschlagen. Dies führt unweigerlich zu einer Reihe von Problemen, auch für die Betreiber der Plantagen. Tiere, denen ihr Lebensraum Regenwald und entsprechende Nahrungsgrundlagen genommen werden, weichen auf andere Standorte aus und fallen mitunter in die Holzplantagen ein.

\section{Javaneraffen fressen in Holzplantagen}

$\mathrm{Zu}$ den robusten Arten, die auf die Plantagen ausweichen, gehört der Javaneraffe (Langschwanzmakak, Macaca fascicularis), der in Südostasien weit verbreitet und häufig ist. Die graubraun bis rötlichbraun gefärbten Tiere fallen durch ihren langen, bis $65 \mathrm{~cm}$ langen

Abb. 1 (Seite 36 links): Vierjährige Plantage mit Acacia mangium.

Abb. 2 (Seite 36 rechts): Fruchtender Zweig von Acacia manguin.

Abb. 3 (oben): Javaneraffe.
Schwanz auf. Unter den Affen ist er die am weitesten nach Süden verbreitete Art. Traurige Bekanntheit haben Javaneraffen durch Einsatz in qualvollen Tierversuchen erlangt.

Javaneraffen halten sich bevorzugt in Wäldern auf, wobei sie in Horden bis etwa 60 Tieren zusammenleben. Sie sind Allesfresser, ernähren sich aber vor allem von Früchten. Mit dem Abholzen der Regenwälder wandern große Gruppen nun zunehmend in Holzplantagen ein, um sich statt von Waldfrüchten von Blättern und Rinde der Plantagenbäume zu ernähren. Aktuell sind auf Sumatra etwa 1,5 Mio. Hektar Holzplantagen, die vornehmlich von den großen einheimischen Zellulose- und Papiergiganten der Zelluloseindustrie betrieben werden, durch Javaneraffen bedroht. Affenhorden fallen vielerorts in Monokulturen aus der nicht heimischen Acacia mangium ein. Die Affen ernähren sich dort von der saftigen Innenrinde der Bäume. Infolge des großen Nahrungsangebots haben sich die recht aggressiven Affenhorden stark vermehrt. Umweltschützer mahnen, dass hier die zerstörte Natur zurückschlägt. 


\section{Absterben der Akazien und Alternativen für die Plantagenbetreiber}

Es gibt keine Mittel, die Affen zu vertreiben. Ein Vergällen der Rinde ist technisch kaum möglich, weil die Affen die Bäume in ihren Kronen schälen. Auch wenn die Akazien nicht unmittelbar durch die Verletzung sterben, werden sie nachfolgend von Wurzelfäule oder von Ceratocystis-Pilzen befallen. Wurzelfäule tötet den Baum innerhalb eines Jahres, von Ceratocystis befallene Akazien gehen bereits nach wenigen Wochen ein.

Die bisher geplante Ernte nach fünf bis sieben Jahren ist für die Plantagenbetreiber zu einer Illusion geworden. Forstexperten betonen, dass die Nachhaltigkeit bedroht sei. Die Plantageneigner müssen jetzt bereits nach vier Jahren abernten und ersetzen die Akazien durch Eucalyptus pellita. Es handelt sich hierbei um eine Art, die bisher nicht geschält wurde, dafür aber geringere Erträge liefert. Auf torfigen Sumpfböden kann sie gar nicht angebaut werden. Auf diesen Böden wurde bisher Acacia crassicarpa für die Plantagen bevorzugt, die aber inzwischen auch schon zu fünfzig Prozent von Wurzelfäule befallen ist. Es ist zu befürchten, dass bei sinkender Produktivität der Holzbedarf aus Plantagen immer schlechter gedeckt werden kann und dementsprechend weiter Wälder abgeholzt werden.

\section{Bundesverdienstkreuz für HERWIG ZAHORKA in Indonesien}

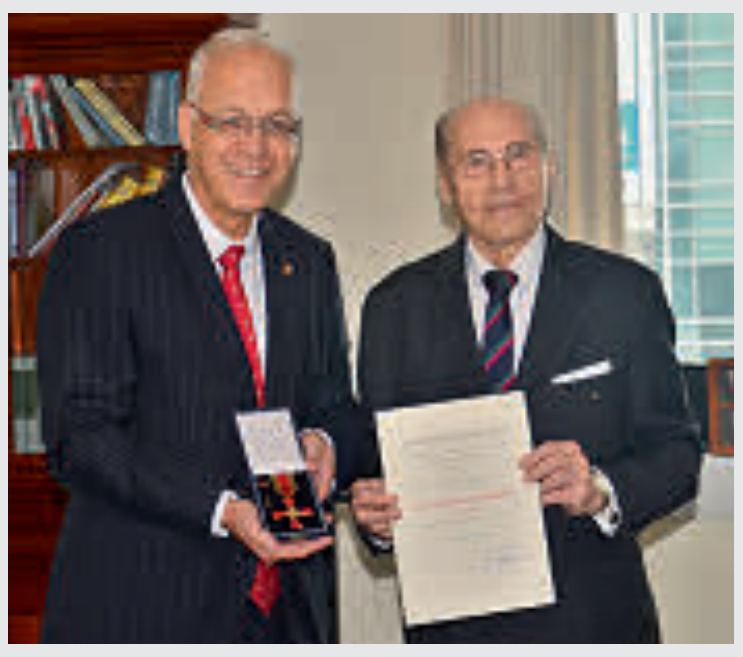

Abb. 4: Herwig Zahorka bei der Verleihung des Bundesverdienstkreuzes.

Der Bundespräsident verlieh Forstdirektor i. R. Herwig Zahorka aus Wiesbaden, seit vielen Jahren Autor für den Palmengarten, „in Anerkennung der um Volk und Staat erworbenen besonderen Verdienste" das Bundesverdienstkreuz am Bande. Der Orden wurde ihm vom deutschen Botschafter in Indonesien, Georg Witschel, in der Botschaft in Jakarta überreicht. ZAHORKA lebt mit einem Forschungsvisum seit vielen Jahren in Indonesien. In seiner Laudatio erwähnte der Botschafter die umfangreiche wissenschaftliche Forschungstätigkeit
ZaHORKas in Indonesien, die in bisher rund 250 Publikationen, einschließlich vier Büchern, ihren Ausdruck fanden. Der Schwerpunkt liegt bei Ethnobotanik, tropischer Dendrologie, allgemeiner tropischer Botanik, Ethnografie und Archäologie. Damit habe er die deutsch-indonesischen Beziehungen auf kulturellen und wissenschaftlichen Gebieten besonders gefördert. Daneben initiierte Zahorka im Jahre 2007 die Errichtung eines deutschen Kulturdenkmals im Botanischen Garten Bogor für den deutschen Botaniker Reinwardt, der 1817 den Garten gegründet hat.

Zur Frankfurter Buchmesse (Gastland: Indonesien) erscheint im Herbst 2015 das von Herwig Zahorka verfasste Buch „, Die Siberuter; mit ihrer steinzeitlichen Religion haben sie den Regenwald erhalten“. ZAHORKA beschreibt einen noch neolithisch lebenden Stamm auf der einsamen indonesischen Insel Siberut mit einer friedlichen, den Regenwald erhaltenden Religion. Diese wird von der Regierung verboten und verfolgt. Die vielen bei den Ritualen benutzten Pflanzen wurden vom Autor botanisch identifiziert und im Buch vorgestellt. Die einzigartige Kultur der Siberuter ist auf dem Weg, zerstört zu werden. 